\title{
Sleep benefits in parallel implicit and explicit measures of episodic memory
}

\author{
Frederik D. Weber, ${ }^{1,3}$ Jing-Yi Wang, ${ }^{1,3}$ Jan Born, ${ }^{1,4}$ and Marion Inostroza ${ }^{1,2}$ \\ ${ }^{1}$ Institute of Medical Psychology and Behavioral Neurobiology, University of Tübingen, 72076 Tübingen, Germany; ${ }^{2}$ Departamento de \\ Psicología, Universidad de Chile, 1058 Santiago de Chile, Chile
}

\begin{abstract}
Research in rats using preferences during exploration as a measure of memory has indicated that sleep is important for the consolidation of episodic-like memory, i.e., memory for an event bound into specific spatio-temporal context. How these findings relate to human episodic memory is unclear. We used spontaneous preferences during visual exploration and verbal recall as, respectively, implicit and explicit measures of memory, to study effects of sleep on episodic memory consolidation in humans. During encoding before 10-h retention intervals that covered nighttime sleep or daytime wakefulness, two groups of young adults were presented with two episodes that were 1-h apart. Each episode entailed a spatial configuration of four different faces in a $3 \times 3$ grid of locations. After the retention interval, implicit spatio-temporal recall performance was assessed by eye-tracking visual exploration of another configuration of four faces of which two were from the first and second episode, respectively; of the two faces one was presented at the same location as during encoding and the other at another location. Afterward explicit verbal recall was assessed. Measures of implicit and explicit episodic memory retention were positively correlated $(r=0.57, P<0.01)$, and were both better after nighttime sleep than daytime wakefulness $(P<0.05)$. In the sleep group, implicit episodic memory recall was associated with increased fast spindles during nonrapid eye movement (NonREM) sleep $(r=0.62, P<0.05)$. Together with concordant observations in rats our results indicate that consolidation of genuinely episodic memory benefits from sleep.
\end{abstract}

Originally, episodic memory has been defined with reference to stored "information about temporally dated episodes or events, and temporal-spatial relations between them" (Tulving 1983). Specific to episodic memory is that an experienced event upon its one-time occurrence becomes bound to the particular temporal and spatial context in which it occurred (Tulving 2002). However, apart from the binding of item memory into spatiotemporal context, the episodic memory concept originating from human research has also emphasized the dependence of episodic memory on autonoetic consciousness during recollection, which refers to a subjective awareness of the self as part of the remembered episode (Tulving 2001, 2002). Because examination of these subjective aspects of episodic memory appears to be suitable only for language-based approaches, research in animals has focused on the core features of episodic memory in terms of a memory for "what" (event) happened "where" (spatial location) and "when" (temporal order of events; Clayton and Dickinson 1998; Clayton et al. 2003), leaving unanswered the question to which extent this memory is truly episodic (Klein 2013; Pause et al. 2013).

There is now ample evidence that sleep benefits the consolidation of memory (Rasch and Born 2013). It has been proposed (Diekelmann and Born 2010) that sleep supports, in particular, the system consolidation of hippocampus-dependent memory which, in the classical view, is declarative memory and comprises episodic and semantic memories (Squire 1992; Diekelmann and Born 2010). According to this concept, slow wave sleep (SWS) promotes the neuronal reactivation of newly encoded hippocampal

\footnotetext{
${ }^{3}$ These authors are joint first authors.

${ }^{4}$ Corresponding author

E-mail jan.born@uni-tuebingen.de

Article is online at http://www.learnmem.org/cgi/doi/10.1101//m.033530.113.

Freely available online through the Learning \& Memory Open Access option.
}

memory representations and thereby not only strengthens them but also stimulates their redistribution to extrahippocampal networks serving as long-term store. Rapid eye movement (REM) sleep might add to consolidation by promoting synaptic consolidation processes which also would enhance nonhippocampal, e.g., procedural, types of memory (Diekelmann and Born 2010).

Although numerous studies in humans have demonstrated that sleep strengthens declarative memory, the effects of sleep on strictly episodic memory and its item-context binding features are less well investigated (Inostroza and Born 2013). There is some evidence that sleep preferentially strengthens context over item memory (Rauchs et al. 2004; Spencer et al. 2006; Lewis et al. 2011; van der Helm et al. 2011). However, others failed (Cairney et al. 2011), and none of these studies specifically examined the binding of an event into spatio-temporal context as a key feature underlying the formation of episodic memory. Notably, effects of sleep on the binding of item memory into spatio-temporal context have so far been directly examined only in one study in rats (Inostroza et al. 2013a). This study revealed sleep to, indeed, be necessary for upholding an integrative episodic-like representation. Rats which remained awake during the 80-min retention interval following encoding did not display any significant signs of episodic-like memory at retrieval testing, and in separate experiments these rats also forgot spatial and temporal context memory.

The present study followed two aims: First, based on the evidence in rats (Inostroza et al. 2013a), a supporting effect of sleep on core features of episodic memory-i.e., the binding of item memory into a spatio-temporal context-should be demonstrated in healthy humans. Second, we aimed at establishing a close link of the findings about the sleep-dependency of episodic-like (C) 2014 Weber et al. This article, published in Learning \& Memory, is available
under a Creative Commons License (Attribution-NonCommercial 4.0 Inter-
national), as described at http://creativecommons.org/licenses/by-nc/4.0/. 
memory in rats to human episodic memory. For this purpose, we adopted a task paradigm in humans that assessed episodic memory, like in rats, based on exploratory preferences (Kart-Teke et al. 2006). Whereas in rats exploratory locomotor behavior is typically used to assess episodic memory, in our human participants we used visual exploration. Importantly, in so doing we established a nonverbal, implicit measure of episodic-like memory in humans, which we tested for its correlation with truly explicit episodic memory recall.

\section{Results}

\section{Implicit episodic memory}

Analysis of variance (ANOVA) of normalized visual exploration time for the different items (faces) during the retrieval phase revealed different patterns depending on whether participants had slept or were awake during the retention interval between encoding and retrieval $\left(F_{(1,27)}=5.52, P=0.026\right.$, for Displaced/ Stationary [Spatial component] $\times$ Old/ Recent [Temporal component] $\times$ Sleep $/$ Wake [Condition]). Separate analyses of the Sleep and Wake groups indicated that only the Sleep group displayed significant episodic memory, i.e., a pattern of visual exploration that matched exploratory preferences in rodents with significant episodic-like memory (Li and Chao 2008; Inostroza et al. 2013a). The episodic nature of the expressed memory integrating temporal and spatial components manifests itself in the interaction between spatial and temporal components of the task $\left(F_{(1,14)}=6.56, P=\right.$ 0.023 , for Spatial component $\times$ Temporal component in a sub-ANOVA on the Sleep group) (Fig. 1B), i.e., a pattern that is primarily characterized by relatively shorter exploration time for the face that is both old-familiar and displaced than would be expected from adding up the spatial main effect (i.e., longer exploration for the displaced than stationary faces) and the temporal main effect (i.e., longer exploration for the old-familiar than recent-familiar faces). The Wake group did not display a significant pattern of visual exploration, i.e., no indication of episodic memory $(P>$ 0.36 , for Spatial component $\times$ Temporal component interaction). Post-hoc $t$-tests between the groups revealed greatest differences for the recent-familiar stationary item for which exploration time, on average, was shortest in the Sleep group (mean \pm SEM, $431.9 \pm 53.00 \mathrm{msec}$ ) but longest in the Wake group (763.57 \pm 98.81 msec, $t_{(27)}=-3.015, P=0.006$, $d=1.11$ ) (Fig. 1B). Spatial and Temporal component main effects were not significant $(P>0.32)$.

The presence of episodic memory in visual exploration patterns at retrieval chance level (zero).

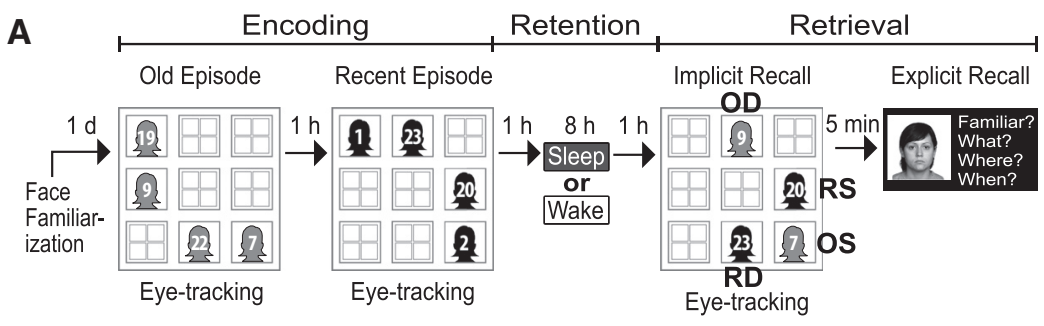

selectively in the Sleep group was confirmed using the "episodic binding" score in which basically the statistical Spatial component $\times$ Temporal component interaction term was used to specifically express the spatio-temporal binding underlying the formation of an episode (see Materials and Methods). Accordingly, significant spatio-temporal episodic binding was only observed in the Sleep group $\left(13.47 \pm 5.25 \%, t_{(14)}=2.56, P=\right.$ $0.023, d=0.66)$ but not in the Wake group $(-6.03 \% \pm 6.49 \%$,

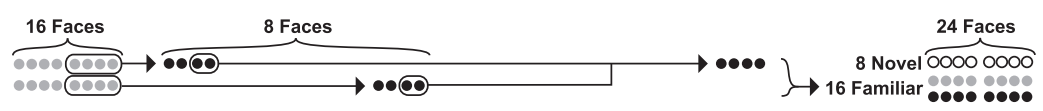

B

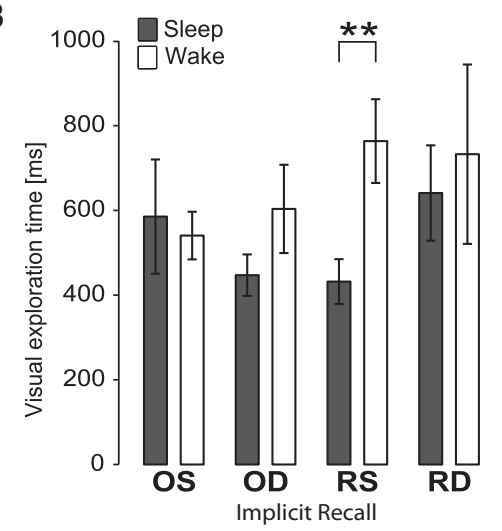

C

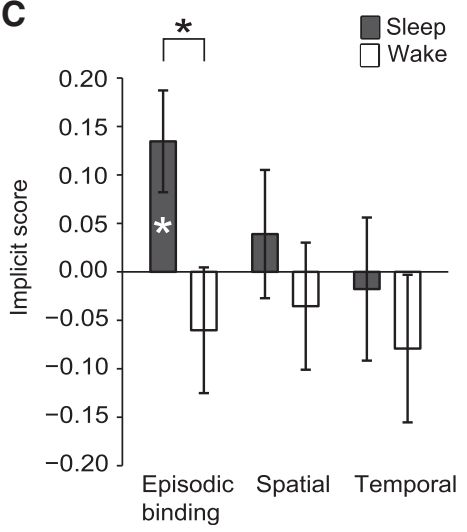

Figure 1. (A) Experimental design. Each session included an encoding phase, a retention interval, and a retrieval phase. The encoding phase comprised two episodes (Old Episode, Recent Episode) 1-h apart, each entailing a specific configuration of four individual faces in a $3 \times 3$ grid of locations. The subsequent 10-h retention interval contained either an 8-h interval of nighttime sleep (Sleep group) or daytime wakefulness (Wake group). The retrieval phase started with implicit recall which was assessed by eye-tracking visual exploration of another configuration of four faces. Two of these faces were from the first (Old) and second (Recent) episode, respectively, and of the two faces one was presented at the same location (Stationary) as during the episode and the other at another location (Displaced) resulting in four stimulus types: Old-familiar Stationary (OS), Old-familiar Displaced (OD), Recent-familiar Stationary (RS), and Recent-familiar Displaced (RD). Implicit recall was followed by explicit verbal recall testing. Face configurations were randomized across episodes and implicit recall. For the figure, individual faces are anonymized by ID-numbers representing one of the total set of 24 faces used in the task (first episode faces_-black; second episode faces_-gray; one gray-scaled example face illustrated for explicit recall). Bottom part illustrates faces used in the different experimental phases: in the face familiarization phase before the experiment proper, subjects were familiarized with 16 faces (gray circles), of which eight faces were used in the encoding phase of the episodic memory task, four in the old episode, and four in the recent episode. For implicit recall testing, four of the faces presented in the episodes of the encoding phase were used, two from each episode. During explicit recall testing 24 faces were presented, i.e., aside from the 16 familiarized faces (eight from episodesblack circles, eight not from the episodes but presented in the face familiarization phase-gray circles), and eight entirely novel faces (empty circles), which also allowed to discrimination between "face recognition" (novel vs. familiar) and "What" memory (familiar in episodes vs. familiar but not in episodes). (B) Mean ( \pm SEM) visual exploration time for each stimulus type, and $(C)$ "episodic binding" scores (indicating spatio-temporal binding in episodic memory) (see Materials and Methods) and separately measures of the spatial and temporal components in episodic memory during implicit recall testing, for the Sleep group ( $n=15$, filled bars) and the Wake group $(n=14$, empty bars). Note, for clarity, absolute rather than normalized exploration time (i.e., exploration time divided by the total time of all looks on a face) is indicated. $\left({ }^{*}\right) P<0.05,\left({ }^{* *}\right) P<0.01$, above bars for difference between Sleep and Wake groups, within bars (in panel $C$ ) for comparison with 
$\left.t_{(13)}=-0.93, P=0.37\right)$, and the positive episodic binding score of the Sleep group also significantly differed from that of the Wake group $\left(t_{(27)}=2.35, P=0.026, d=0.87\right)$ (Fig. 1C). Scores formed separately for the spatial and temporal components of episodic memory (reflecting the statistical Spatial and Temporal main effects) failed to reach significance in both the Sleep and Wake groups and also did not differ between the groups (spatial score-Sleep, $3.90 \pm 6.62 \%$; Wake, $-3.54 \pm 6.56 \%, t_{(27)}=0.80$, $P=0.43$; temporal score-Sleep, $-1.78 \pm 7.38 \%$; Wake, $-7.91 \pm$ $\left.7.62 \%, t_{(27)}=0.58, P=0.57\right)$.

For both experimental groups, control analyses excluded any transition effects, i.e., exploration time was not influenced depending on whether or not during the encoding phase a certain grid location was occupied by an item in both episodes (Sleep, $t_{(13)}=-0.18, P=0.86$; Wake, $t_{(12)}=-0.12, P=0.91$; comparison between groups $t_{(25)}=-0.046, P=0.96, d=0.02$ ).

\section{Explicit episodic memory}

Explicit recall of episodic memory ("what-where-when" memory) was assessed after implicit retrieval measurement, and determined by the percentage of (all possible) faces that the participant correctly identified as occurring in one of the episodes ("what") and for which he also identified the correct episode ("when") and the location ("where") at which it occurred (see Materials and Methods). Explicit episodic recall was above chance in both groups (Sleep $P<0.001$, Wake $P=0.025$, Mann-Whitney test) but differed between the conditions, with performance being distinctly better in the Sleep than Wake group $(41.79 \pm 6.94 \%$ vs. $17.86 \pm 6.51 \%, P=0.005, r=0.51$ ) (Fig. $2 \mathrm{~A}$ ). Higher recall performance in the Sleep than Wake group was confirmed in an analysis restricted to faces that were not involved in implicit recall $(P<0.022)$ excluding a biasing influence of prior implicit recall testing. In an exploratory ANOVA, episodic memory recall did not differ between faces of the first and second episode $(P>$ 0.18 , for respective Episode main effect and Episode $\times$ Sleep/ Wake interaction).

Separate analysis of the "what" component (number of faces correctly identified as belonging to one of the two episodes expressed as percentage of episode faces that were correctly recognized as familiar and belonging to one of the two episodes) revealed performance well above chance in both conditions

A
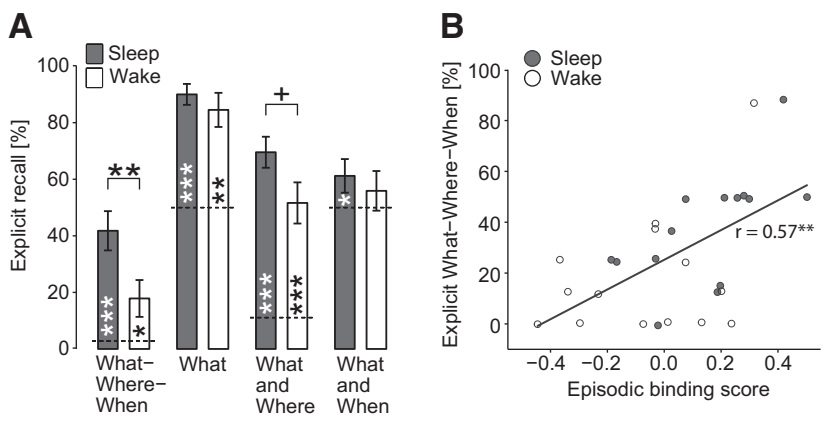

Figure 2. ( $A$ ) Mean ( \pm SEM) explicit recall of episodic "What-WhereWhen" memory and of subcomponents ("What," "What and Where," "What and When") during the retrieval phase for the Sleep $(n=15$, filled bars) and Wake groups ( $n=14$, empty bars). $\left({ }^{+}\right) P<0.1,\left(^{*}\right) P<$ $0.05,\left({ }^{*}\right) P<0.01,\left(^{* *}\right) P<0.001$, above bars for difference between groups, within bars for comparison with chance level (dotted line). (B) Pearson product-moment correlation between implicit episodic memory (Episodic binding score) and explicit episodic memory recall (WhatWhere-When), across the Sleep (filled circles) and Wake groups (empty circles, $n=28$; data from one Sleep subject was excluded due to ceiling, $100 \%$, explicit recall performance). $\left(^{*}\right) P<0.01$.
(Sleep, $90.0 \pm 3.70 \%, P<0.001$; Wake, $84.52 \% \pm 6.03 \%, P=$ $0.002)$ but without a significant group difference $(P=0.43)$. The proportion of recalled faces (i.e., "what" component) for which the place was correctly recalled ("what and where") was above chance in both groups (Sleep, $69.55 \pm 5.48 \%$; Wake, $51.62 \pm$ $7.28 \%$; both $P<0.001$ ), and was marginally (but not significantly) greater in the Sleep group $(P=0.057)$. The proportion of recalled faces for which the episode was correctly recalled ("What and When") was above chance only in the Sleep group $(61.19 \pm 5.95 \%, P=0.040)$, but failed to reach significance in the Wake group $(55.90 \pm 7.01 \%, P=0.21$; for the difference between groups, $P=0.57)$. Interestingly, restricting the analysis of these items (for which "What and When" was correctly recalled) to only those for which the spatial component ("WhatWhere") was not correctly recalled yielded a significantly better recall for the Wake than the Sleep group $(12.71 \pm 3.91 \%$ vs. $30.07 \pm 6.21 \%, P=0.016)$ suggesting that temporal processing considered in isolation might be superior in the wake state. Finally, an overall analysis of faces correctly recognized as familiar, which included also those faces presented only during the familiarization phase, indicated that both the Sleep and Wake groups displayed close to ceiling object-recognition performance, with no differences between groups (Sleep, $91.67 \pm 3.64 \%$; Wake, $94.64 \pm 2.61 \%, P=0.90)$.

\section{Correlation analyses}

We calculated correlations between implicit and explicit measures of episodic memory and, for the Sleep group, between recall measures and sleep parameters. The implicit episodic binding score inferred from visual exploration was significantly correlated with explicit episodic memory recall ("What-Where-When") across both groups $(r=0.57, P=0.002)$ (Fig. 2B), which was mainly driven by the sleep group (Sleep, $r=0.63, P=0.017$; Wake, $r=$ $0.21, P=0.47)$. There was no correlation between separate implicit and explicit scores of spatial or temporal components of episodic memory $(P>0.30)$.

The Sleep group displayed normal overnight sleep during the retention interval (see Table 1 for a summary of sleep parameters). Correlation analyses revealed a consistent pattern of moderate associations, in particular between NonREM sleep processes and implicit measures of episodic memory. Thus, the episodic binding score showed a positive correlation with time in NonREM sleep $(r=0.68, P=0.007)$ and, in parallel, consistent correlations with centro-parietal (fast) spindle counts during NonREM sleep $(r=$ $0.62, P=0.017$, for average spindle count across central and parietal electrodes) (Fig. 3). In further exploratory analyses, the separate temporal memory component score showed negative correlations with time in stage 2 NonREM sleep $(r=-0.71, P=$ $0.004)$ and fast spindles counts over centro-parietal areas $(r=$ $-0.57, P=0.03$, for average count across electrodes). There were no consistent correlations with EEG power in the frequency ranges of interest with the exception of a negative correlation between "What" memory and NonREM EEG activity in the 0.5- to $8-\mathrm{Hz}$ range that was highest for the delta band $(P<0.0001$, $r=-0.88)$. Explicit episodic memory recall did not show any significant correlation with sleep measures, and there was also no consistent association between memory measures and REM sleep parameters.

\section{Memory for word-pair associates, vigilance, and mood}

Between the experimental episodes of the encoding phase, participants learned two lists of word-pairs, i.e., a control task of declarative memory for which beneficial effects of sleep are well established (e.g., Plihal and Born 1997). As expected, recall of the word-pairs tested at the end of the retrieval phase was 
Table 1. Sleep parameters for experimental night of the Sleep group

\begin{tabular}{lc}
\hline Sleep stages & Time in minutes \\
\hline TST & $467.29 \pm 6.01$ \\
Sleep onset & $14.21 \pm 3.52$ \\
Wake & $15.93 \pm 8.10$ \\
Stage 1 & $14.43 \pm 2.82$ \\
Stage 2 & $239.29 \pm 11.47$ \\
Stage 3 & $40.36 \pm 2.19$ \\
Stage 4 & $55.25 \pm 7.98$ \\
SWS latency & $14.75 \pm 1.50$ \\
SWS & $95.61 \pm 7.82$ \\
REM latency & $113.46 \pm 10.89$ \\
REM & $98.64 \pm 6.77$ \\
\hline
\end{tabular}

Data are means \pm SEM, $n=14$. Total sleep time (TST) and time in different sleep stages, sleep onset latency (with reference to lights off) and latency for slow wave sleep (SWS) and rapid eye movement (REM) sleep with reference to sleep onset.

significantly better in the Sleep than Wake group $(99.21 \pm 2.80 \%$ vs. $\left.91.13 \pm 2.67 \%, t_{(27)}=2.09, P=0.047, d=0.78\right)$.

Vigilance was assessed by the Psychomotor Vigilance Task (PVT) before each episode during the encoding phase and before implicit recall during the retrieval phase, but did not differ between groups (Sleep vs. Wake: before first episode $291.35 \pm 32.41$ msec vs. $286.46 \pm 20.34 \mathrm{msec}$; before second episode, $296.35 \pm$ $21.72 \mathrm{msec}$ vs. $291.92 \pm 27.47 \mathrm{msec}$; before implicit recall, $291.73 \pm 24.00 \mathrm{msec}$ vs. $285.68 \pm 23.11 \mathrm{msec}$; all $P>0.50$ ). There were also no differences between groups in subjective sleepiness assessed by the Stanford Sleepiness Scale (SSS) (Sleep vs. Wake: before first episode $3.25 \pm 1.14$ vs. $2.84 \pm 0.99$; before second episode, $3.5 \pm 1.17$ vs. $2.92 \pm 1.12$; before implicit recall $2.50 \pm 0.80$ vs. $1.92 \pm 0.76$; all $P>0.10$ ), and in mood, assessed by the Positive Affect Negative Affect Scale (PANAS) (Sleep vs. Wake group: before encoding phase, Positive Affect $24.43 \pm$ 1.25 vs. $27.62 \pm 1.8, P>0.40$, Negative Affect $12.93 \pm 0.91$ vs. $12.54 \pm 0.55, P>0.76$; before retrieval phase, Positive Affect $26.16 \pm 1.52$ vs. $29.36 \pm 1.64, P>0.23$, Negative Affect $12.54 \pm$ 0.69 vs. $11.93 \pm 0.35, P>0.66)$.

\section{Discussion}

We report novel evidence indicating that sleep in humans strengthens the binding of an item memory into spatio-temporal context which is a core feature of episodic memory. Importantly, we assessed episodic memory implicitly by visual exploration times, and explicitly by verbal recall, and for both measures sleep compared to wakefulness produced a more than twofold increase in strength of episodic binding. Although explicit assessment suggests episodic binding is present after sleep and wakefulness, implicit assessment indicated above-chance episodic binding only if subjects slept after encoding. Compared with the distinct effect on episodic binding, sleep had only minor effects on separate implicit or explicit retrieval measures of "What," "Where," and "When" components of the encoded episodes. To immediately support a fresh episodic memory might represent a basic component of sleep's function in memory processing.

We established a novel task that allowed for assessing truly episodic memory in humans in both ways, i.e., implicitly, using visual exploration, and explicitly, using verbal recall. The task design originated from previous studies that employed behavioral exploration preferences to investigate episodic-like memory in rodents (Dere et al. 2006; Kart-Teke et al. 2006, 2007; DeVito and Eichenbaum 2010; Davis et al. 2013a,b; Inostroza et al. 2013a,b). The episodic nature of our task was further enhanced by using unique faces that were presented in a unique spatio-temporal context. To reduce emotionality, we used faces with a neutral expression that, in addition, were familiarized before the experiment proper. Emotionality has been considered a feature inherent to episodic memory and, indeed, is a critical factor determining persistence of episodic memory (Libkuman et al. 2004; Dere et al. 2010; Pause et al. 2013). Nevertheless, we preferred to make the experienced episodes relatively neutral, because in this way visual exploration was expected to be determined predominantly by novelty, preventing that emotional aspects in the stimulus configuration masked memory-guided visual exploration.

Implicit memory was successfully indicated in the task by visual exploration time, specifically the time participants spent looking at a particular face at their first looks on a face, with the exploration times being characteristically enhanced when a face at retrieval testing is encountered in a conflicting spatio-temporal context relative to the previously encoded episodes. Thus, visual exploration time is longer for faces spatially displaced than for (stationary) faces presented at the same location as during encoding, and in parallel with temporal conflict, exploration time is longer for faces that belong to the older compared to the more recently encoded episode. A consistent exploration pattern for the faces with conflicting contexts indicates associated spatial and temporal memory. Crucially, episodic binding of an event together with spatial and temporal context components expresses itself in an interaction of spatial and temporal memory effects for a unique face, as derived from the exploration pattern, rather than in a mere additive effect of both components. This interaction between "when" and "where" effects on visual exploration time indicates that the gain in exploration time for faces that are both old and displaced (i.e., OD) is less than would be expected from adding up spatial and temporal main effects. It is this interaction expressing itself in a relatively reduced exploration of OD items that has been consistently revealed as an indicator of episodic-like memory in rodent studies (e.g., Kart-Teke et al. 2006, 2007; Inostroza et al. 2013a), and that provides a valid measure of an integrated rather than separated retrieval of spatial and temporal context, i.e., of contextual binding as a hallmark of episodic memory (Clayton et al. 2003, but see also Place et al. 2012).

Semantic memory can mask the assessment of episodic memory and, although our subjects were not instructed to learn anything, the encoding phase comprising three consecutive runs through each episode might have triggered semantic memory formation (Pause et al. 2013). However, simple models of familiarity-based recall, where trace strength is reflected in memory strength, would predict generally better recall performance for the recent than the older episode. This is not supported by our results as there were no significant differences in this direction

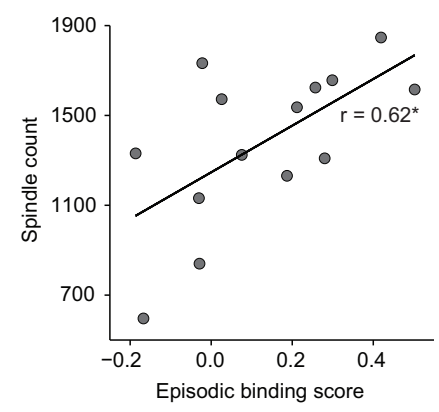

Figure 3. Pearson product-moment correlation in the Sleep group $(n=14)$ between fast sleep spindle counts during NonREM sleep and implicit episodic memory (Episodic binding score). $\left(^{*}\right) P<0.05$. 
for any of the implicit or explicit memory measures. Explicit "what-where-when" memory was even better for the first than second episode in the Wake group, possibly reflecting proactive interference which was annulled by sleep (Abel and Bäuml 2013). Also, we controlled that our implicit measure of episodic memory is, indeed, item specific, i.e., it is only sensitive to a specific face ("what") and not to any face in a shared spatio-temporal context. This was indicated by calculating a "transition score" which excluded that mere spatial overlaps regarding the occupation of a grid location between the first and second episode significantly contributed to our episodic memory measure. Finally, implicit assessment of memory on only half of the items involved in each episode allowed us to control whether implicit recall biased subsequent assessment of explicit memory. Although such bias cannot be entirely ruled out, explicit "what-wherewhen" memory for the faces used in implicit recall testing showed no difference from memory for items that were not used.

Tulving (2002) considered episodic memory recall a capacity that involves the ability to "mentally time travel" and re-experience specific events, and thereby relies on a sense of self and conscious awareness that the experience occurred in the past. Although this definition reflects the phenomenological aspects of episodic memory in humans, it exclusively relies on the verbal report of subjective experiences. However, it prevents its investigation in animals. The absence of any objective behavioral measure for episodic memory is also not conducive to a rigorous scientific investigation of this kind of memory in humans (Allen and Fortin 2013). Here, this issue is addressed. A major advantage of our implicit memory measure is that it allows for examining episodic memory in nonverbal humans, i.e., infants, and also for comparisons across species. Although not specifically scoring for episodic binding, studies in rodents using exploration behavior in an analogous task design revealed an episodic-like pattern strikingly similar to that observed here for human visual exploration (Dere et al. 2006; Kart-Teke et al. 2006, 2007; Davis et al. $2013 a, b)$. Furthermore, those studies showed that this episodiclike memory exploration pattern is crucially dependent on hippocampal function (DeVito and Eichenbaum 2010) and sleep (Inostroza et al. 2013a). The latter observation concurs with the present study where the episodic-like memory exploration pattern was also robustly expressed only in the participants of the Sleep group. Thus, the present findings constitute a strong link between sleep-dependency in human episodic memory and episodic-like memory in rodents. Pause et al. (2010) took an approach to explore episodic memory-like exploration patterns in humans comparable to ours. However, rather than on visual exploration they relied on explorative button presses as a nonverbal measure, and subjects were instructed to learn the episodes at encoding. At a recall test $24 \mathrm{~h}$ later, they found nearly significant spatial and temporal main effects, however no cues for episodic binding, suggesting that visual exploration might be more sensitive to preferences promoted by implicit memory than exploratory motor behaviors.

Sleep's function for declarative memory has been conceptualized to be an active system consolidation process rather than a passive protection against interference (Stickgold 2005; Diekelmann and Born 2010; Lewis and Durrant 2011; Inostroza and Born 2013; Rasch and Born 2013). According to this concept, an episode is encoded in both hippocampal and extrahippocampal networks, whereby the hippocampus preferentially encodes aspects binding items into their unique spatio-temporal context. During subsequent SWS, hippocampal portions of the representation are repeatedly reactivated to support an immediate strengthening of hippocampal traces and also redistribution toward preferential storage of information in extrahippocampal networks. This redistribution entails a transformation of represen- tations toward more decontextualized schema-like representations (Marr 1971; Frankland and Bontempi 2005; Diekelmann and Born 2010; Winocur et al. 2010). The EEG slow oscillation and fast $(12-15 \mathrm{~Hz})$ spindles are considered hallmarks of the consolidation processes, in as much as the slow oscillation appears to synchronize hippocampal memory reactivations and accompanying sharp wave-ripples with the occurrence of spindles, thus allowing for the formation of spindle-ripple events as a mechanism supporting the transfer of reactivated memory information toward extrahippocampal circuitry (Mölle and Born 2011; Mölle et al. 2011). Consistent with this view, here we found a robust association between episodic memory recall and spindle counts during post-encoding NonREM sleep. Together with numerous previous studies showing similar correlations between sleep spindle activity and the retention of declarative as well as procedural memory (e.g., Gais et al. 2002; Tamaki et al. 2009; Barakat et al. 2011; Wilhelm et al. 2011; Rasch and Born 2013), this observation does not only underline the importance of spindles for memory processing in general but also points to a specific function of spindles in enhancing spatio-temporal integration in a memory, which might be conveyed via a direct impact on hippocampal networks (Clemens et al. 2007). The association with spindle activity occurring selectively for implicit rather than explicit episodic memory recall is difficult to explain in this context; it might be that the implicit measure is more sensitive, capturing variability in recall of hippocampal memories to a greater degree than the explicit recall measure.

Implicit but not explicit recall showing robust correlation with sleep spindles raises the question whether the two recall measures access the same representation, although via different retrieval pathways, or whether there exist distinct implicit and explicit representations that happen to correlate significantly because they encode for the same experience. That the wake group showed significant explicit episodic memory, but chance level performance for implicit episodic memory recall, could be taken as a hint for two different representations being accessed in the tests. This assumption would be further supported if implicit and explicit episodic memory underwent different transformations across sleep. However, as we did not assess recall before the retention interval, sleep-induced memory transformation could not be examined here. Nevertheless, the effects of sleep compared with wakefulness, being surprisingly in parallel for implicit and explicit measures of episodic memory, speak in favor for a common representation underlying both types of recall measures which, indeed, share the essential features of episodic experience, but the autonoetic consciousness that is produced only during explicit recollection.

\section{Materials and Methods}

\section{Participants}

Participants were healthy, nonsmoking, and native-speaking volunteers recruited from the campus of the University of Tübingen. They were randomly assigned to either the Sleep group ( $n=15$, nine men) aged (mean \pm standard deviation) $23.72 \pm$ $3.14 \mathrm{yr}$, or the Wake group $(n=14$, seven men, age $=24.25 \pm$ $5.08 \mathrm{yr}$ ). They had normal or corrected to normal vision and did not take any medication at the time of the experiments. They had a normal sleep-wake rhythm and were not on any night shifts during the $6 \mathrm{wk}$ preceding the experimental session. Participants were instructed to keep their regular sleep schedule, abstain from caffeine- and alcohol-containing drinks for at least $3 \mathrm{~d}$ prior to and on the days of the experiments. Prior to the experiment proper, participants of the Sleep group spent one habituation night in the sleep laboratory. Subjects gave written informed consent before participating and the study was approved by the local ethics committee. 


\section{Design and procedures}

The experiments were performed according to a between-groups design, including a Sleep group and a Wake group. For each group, the experiment consisted of an encoding phase followed by an $\sim 10$-h retention interval, followed by a retrieval phase (Fig. 1A). The encoding phase comprised the encoding of two experimental episodes, which were separated by $1 \mathrm{~h}$. The retrieval phase included an implicit recall followed by an explicit recall of the materials learned in the encoding phase. For subjects of the Sleep group, the encoding phase took place between 8:15 pm and 10:45 pm, and the retrieval phase between $8 \mathrm{am}$ and 9:30 am. Sixty minutes after the encoding phase, they went to bed (lights off) for an 8-h sleep period. The retrieval phase started 60 min after awakening. For the Wake group, the encoding phase took place between 7:15 am and 11:15 am, and the retrieval phase between 6:15 pm and 9:15 pm. During the wake interval, the subjects followed their usual activities outside the laboratory. They were not allowed to engage in stressful mental and physical activities. Activity during the retention interval was measured by a wristwatch (Actiwatch 2, Philips). Moreover, subjects provided a report about their activities during this time when they returned to the lab.

To confirm regular declarative memory benefits from sleep, a standard paired words associate learning task (two lists of 40 wordpairs) was used which in previous studies proved sensitive to the effects of sleep (Plihal and Born 1997; Ngo et al. 2013). Lists were learned to a criterion of 24 correctly recalled words (cued recall). In the encoding phase, one list was learned $20 \mathrm{~min}$ after presentation of the first episode of the episodic memory task, and the other 20 min after presentation of the second episode. In the retrieval phase, cued word recall was tested 10 min after episodic memory retrieval was completed.

Subjects were familiarized with the face stimuli used in the episodic memory task $1 \mathrm{~d}$ (in two cases $2 \mathrm{~d}$ ) before the experiment proper, to avoid that the use of novel faces would distract the subject's attention from the spatio-temporal features of the task. To control for vigilance, before this familiarization phase as well as before the encoding phase, in between the two episodes, and before retrieval testing the Psychomotor Vigilance Test (PVT, 5 min) and the Stanford Sleeping Scale (SSS) were administered. To measure current mood, the Positive Affect Negative Affect Scale (PANAS) (Watson et al. 1988; Krohne et al. 1996) was given before the encoding and retrieval phases.

\section{Episodic memory task}

The encoding phase of the episodic memory task comprised the presentation of two episodes separated by $1 \mathrm{~h}$ during which subjects engaged in standardized activities (PVT, word-pair associates learning, filling in questionnaires, etc.). During each episode, participants were presented on a screen with a specific spatial configuration of four different faces arranged in a $3 \times 3$ grid of possible locations (with the center location of the grid left always empty) (Fig. 1A).

During the retrieval phase, implicit spatio-temporal recall performance was assessed by eye-tracking. For this purpose subjects were presented with another configuration of four faces used in the encoding phase (see below for the specific characteristics of this configuration to enable testing of implicit episodic memory). Participants were kept unaware during encoding and implicit recall testing that the task aimed at testing memory, but instead were told that attention was measured, and were instructed to attend to the presented stimulus configurations.

Spontaneous preference in visual exploration times was used as measure of memory, analogous to exploration preferences in rats; i.e., relatively longer fixation of a new than familiar face indicates item memory; relatively longer fixation of a familiar face presented at a new than at an old location indicates spatial memory, and relatively longer fixation of a face from the first than second episode indicates temporal memory (Ennaceur and Delacour 1988; Ennaceur and Meliani 1992; Mitchell and Laiacona 1998). Five minutes after implicit recall, explicit recall was measured by asking the subject successively whether a certain face occurred in one of the two episodes, and if so in which episode and at what grid location it occurred.

\section{Stimuli and stimulus presentation}

Stimuli were 24 colored frontal images of natural female faces with neutral expression, placed on a white background (taken from the FACES database [Ebner et al. 2010]). Faces were randomized across subjects. Only female faces were used to reduce response variability (Penton-Voak et al. 1999).

Stimuli were presented on a monitor (ASUS Model VE248H, 24 -in, $16: 9,1920 \times 1080 \mathrm{px})$ and controlled using the software Presentation (Neurobehavioral Systems, version 15.1). Subjects sat in a comfortable position with an eye distance of $\sim 60 \mathrm{~cm}$ in front of the screen, with their head leaning against an individually adjusted headrest on the back of the chair. To improve eyetracking data during the episodic memory task, subjects were instructed not to move their head too much and to move only their eyes for visual exploration, but not head and neck.

During the familiarization phase before the experiment proper, subjects were presented with 16 faces (in random order) in five separate runs, separated by 30 -sec breaks. Faces were presented one at a time with a 2-sec interstimulus interval. Immediately after stimulus onset, the face started moving smoothly for $1 \mathrm{sec}$ (a minimum of $450 \mathrm{px}$ ) with the start and stop positions randomly chosen for each face presented on the screen. Then, the face rested for $7 \mathrm{sec}$ on the stop position. One second before onset of each stimulus, a voice (from a speaker) signaled "look now" (German: "Schau mal"). Before the task, subjects were instructed to merely "pay attention" to the presented stimuli. The presentation scheme aimed to minimize any spatial interference with the face presentation during the episodic memory task using all available presentation space. To further avoid context interference, face familiarization took place in another room on another screen.

On the episodic memory task, the faces were presented on a $3 \times 3$ grid, with the grid location designed as windows of a house. Each episode started with indicating to the subject on the screen the episode (" 1 " or " 2 "); then the house with "closed windows" (gray framing without faces) was shown for $15 \mathrm{sec}$, and the subject was to fixate with his/her eyes a cross appearing in the center window (which was not used for face presentations). Once the fixation cross was fixated for $100 \mathrm{msec}$, it disappeared. During this time, instructions appeared on the screen, to concentrate and to explore in which window which face appeared (German: "Erkunden Sie, in welchen Fenstern welche Personen sind!"). Thereafter, with a delay of $50 \mathrm{msec}$, presentation of faces started as soon as the participant had fixated the fixation cross for 100 msec. Face presentation comprised two phases. In the "overview phase," all four face stimuli were shown on their respective location simultaneously for $15 \mathrm{sec}$. In the second "eye-trigger phase," the four faces were covered by "closed" windows and the specific face did not appear until the participant triggered its presentation by looking at the respective closed window area for at least 200 msec. The triggered face was then shown in the respective window. Presentation was discontinued as soon as the subject had looked at the respective stimulus area for a total of $7 \mathrm{sec}$ (regardless of whether or not the gaze was intermittently directed away from the face location) which assured optimal control over the stimulus during encoding. Presentation of each face could be triggered only once, and the phase was completed when all four faces were discovered, i.e., triggered by the participant. During each episode the sequence of overview phase and eye-trigger phase was run three times in an identical manner.

Implicit recall testing consisted of only one overview phase. It involved a configuration of four familiar faces of which two were previously presented in the first ("old-familiar") and the other two in the second episode ("recent-familiar"). Of these, one was presented at the same location ("stationary") as during the respective encoding and the other was presented at another (new) location ("displaced") which was not occupied during the respective episode at encoding. This gives rise to four stimuli types: Old-familiar Stationary (OS), Old-familiar Displaced 
(OD), Recent-familiar Stationary (RS), and Recent-familiar Displaced (RD). Thus, during implicit recall testing only faces that occurred during one of the two episodes of the encoding phase were presented, and no others. Although the face configuration during implicit recall testing was randomized, there were specific rules to position the stimuli that were mainly introduced to minimize potentially confounding effects on exploration time arising from the fact that a certain location could have been occupied by a face in just one episode or both episodes during encoding, and to exclude face-specificity of effects. Thus, the OS face was always on a location that had been occupied during both episodes, with the face from the second episode on this location not being used for implicit recall testing. The RS face was on a location that was empty during the first episode. The OD face was also on a location that was not occupied during the first episode, but was occupied by the face chosen for RD in the second episode. The RD face was then on a location that was not occupied during the second episode, but was occupied in the first episode with a face not used for implicit recall testing. Both episodes shared the location of the OS and another location presenting a face not presented at implicit recall testing. The center location of the grid was not used for face presentation to assure a similar distance of all faces from the center. Face presentation during implicit recall testing lasted between 30 and $60 \mathrm{sec}$, and within these margins was stopped (with a delay of $3 \mathrm{sec}$ ) as soon as looking time for each face was $>3 \mathrm{sec}$ for each of the four face areas.

\section{Eye-tracking and implicit memory measurement}

Eye movements were tracked using a remote system (Eye-Follower 2.0, interactive minds, tracking rate of $60 \mathrm{~Hz}$ on each eye). Eye-tracking was individually calibrated before each episode and before implicit recall testing. During calibration a colored filled circle traveled to one of 13 different calibration points, rested on each point until fixation of that position was detected by the eyetracker, and then moved to the next calibration point. During calibration, the participant also received feedback about fixation performance accuracy. The calibration procedure was repeated until the assessed accuracy and precision reached a criterion of $<0.6^{\circ}$ deviation from five distributed test targets.

Eye-tracking analyses were restricted to the dominant eye as determined by the hole-in-the-card test (Dolman method) (Cheng et al. 2004). Fixations were detected using a $0.8^{\circ}(30 \mathrm{px})$ visual angle gaze deviation threshold from the Euclidean centroid of the ongoing fixation and a minimum fixation duration of 100 msec (six samples), after removing artifacts (due to blinking, eyeocclusion, etc.). Visual exploration time of faces was measured based on "looks." A look on a face was considered the time the dominant eye was within the face's grid location, i.e., the gray frame surrounding a face, without leaving it. A look contained at least one fixation. Then, the visual exploration time spent on each face was determined by dividing the total time of all looks on a face by the number of looks on that face. Looks were sampled only up to the time point when all faces of the configuration had received at least one look.

\section{Implicit memory}

For determination of implicit memory, data from the first 30 sec of recordings were used. To reduce interindividual variability, visual exploration time for each face was normalized by dividing exploration time for the face by the sum of exploration time across all four faces (using absolute exploration times did not essentially change results reported here). Implicit episodic memory was defined by an "episodic binding" score assessing binding, i.e., integrated temporal and spatial aspects of an episode, as it manifests itself in the interaction between spatial exploration preference (i.e., longer exploration time for the "displaced" than "stationary" faces) and temporal exploration preference (i.e., longer exploration time for the "old" than "recent" faces). Specifically, based on corresponding studies of behavioral exploration in rodents (Kart-Teke et al. 2006, 2007; Inostroza et al. 2013a) this spatiotemporal interaction is expected to expresses itself in a gain of vi- sual exploration time for an item that is both old and displaced (i.e., OD) which is distinctly smaller than would be expected from merely adding up spatial and temporal main effects. The episodic binding score was calculated as follows: [(OS+RD) - (OD + $\mathrm{RS})] /[\mathrm{OS}+\mathrm{OD}+\mathrm{RS}+\mathrm{RD}]$, where OS, OD, RS, and RD represent the visual exploration times for the old stationary, old displaced, recent stationary, and recent displaced faces, respectively. Because we hypothesized episodic memory consolidation produces longer visual exploration time for the OS and RD stimuli, the scores were defined such that values were positive for enhanced preference of those objects. Note, this score of episodic memory binding as defined by the interaction between spatial and temporal exploration preferences reflects the integrative assessment of both spatial and temporal information.

As an approach to infer spatial memory separate from temporal memory and vice versa, that is not reflected in the episodic binding score, we additionally calculated two different scores. The spatial score $[(\mathrm{OD}+\mathrm{RD})-(\mathrm{OS}+\mathrm{RS})] /(\mathrm{OS}+\mathrm{OD}+\mathrm{RS}+\mathrm{RD})$ indicated enhanced preference of displaced (OD, RD) over stationary (OS, RS) faces; the temporal score [(OS + OD) - (RS + RD)]/ $(\mathrm{OS}+\mathrm{OD}+\mathrm{RS}+\mathrm{RD})$ indicated enhanced preference of faces from the first episode (OS, OD) over that of the second (RS, RD). To statistically confirm presence of memory, scores were compared with chance level (zero) using two-tailed one-sample $t$-tests.

To examine if exploration time was influenced depending on whether or not during the encoding phase a certain grid location was occupied by a face in both episodes, in control analyses we compared exploration time for faces in the second episode between those that shared (sharing) and those that did not share (nonsharing) the location with another face in the first episode, using the score (sharing - nonsharing)/(sharing + nonsharing). Significance (against zero) of this "transition" score indicated that a face of the second episode which shared its location with a face from the first episode was preferentially explored over second episode faces not sharing their location with a first episode face. The analysis was restricted to the first 15 -sec interval of the second episode. Two participants (one Sleep, one Wake) were excluded from this analysis due to insufficient eye-tracking data on all four objects.

\section{Explicit memory recall}

For explicit recall testing, all 24 faces (eight presented during the episodes and during the familiarization phase, eight presented only during the familiarization phase, and eight completely novel) were presented consecutively (in random order) and the subject had to indicate (with no time limit, by mouse clicks on respective response text to questions presented on the screen) whether the face was new or familiar (object recognition); and if familiar, whether it occurred in the first or second episode (temporal "what-when" memory) or in none of the two episodes (i.e., was presented only during the familiarization phase), and whether it occurred in one of the episodes, at what grid location (spatial "what-where" memory). For the latter question, the grid was presented and the subject indicated the remembered location per mouse click. For each answer, confidence $(0 \%-100 \%$ certainty) was rated immediately afterward. Subjects were trained on the recall procedure, using a dummy face, right before testing. In a final separate recall test, two grids were presented and the subject was asked to indicate which grid locations were occupied by faces during the first and second episode (spatial "where-when" memory).

Explicit episodic memory was determined by the percentage of faces that were correctly identified as occurring in one of the episodes (i.e., "what"), and for which the subject also correctly indicated the episode (i.e., "when") and the grid location (i.e., "where") it occurred, minus the locations for which the subject in the final separate recall test had forgotten that they were occupied with any face, with the number of episode faces correctly identified as familiar set to $100 \%$. The chance level for this score was $2.78 \%$ (i.e., the chance to correctly recognize eight out of 16 familiar faces, one of two episodes, one of nine grid locations, for eight faces). 
In addition, we separately assessed the "what" component of episodic memory as the percentage of episode faces that were correctly recognized as familiar and belonging to one of the two episodes, with the number of episode faces correctly identified as familiar set to $100 \%$, The chance level for this score was $50.0 \%$ (eight of a total of 16 familiar faces). A general face recognition score was defined by the percentage of faces correctly identified as familiar (including those eight faces not used in the episodic memory task), with a chance level of $66.7 \%$ (16 familiar out of 24 faces). Finally, we calculated the percentage of correctly recognized faces for which also the episode ("what and when") or for which also the location ("what and where") was correctly recalled. The latter two scores were calculated additionally for only the cases where the "where" and "when" components, respectively, were not correctly remembered. The presence of explicit memory above chance level was assessed using one-tailed, one-sample $t$-tests or a nonparametric equivalent. Confidence ratings were not considered in the analyses.

\section{Sleep recordings, EEG analyses, and spindles}

To evaluate sleep in the Sleep group, polysomnographic recordings were performed, including EEG recordings from $\mathrm{Fz}, \mathrm{F} 3, \mathrm{~F} 4$, $\mathrm{Pz}, \mathrm{P} 3, \mathrm{P} 4, \mathrm{Cz}, \mathrm{C} 3, \mathrm{C} 4$ (according to the 10-20 system) with linked reference electrodes attached to the mastoids. An electrode at Fpz served as ground. Electrode impedance was $<5 \mathrm{kohm}$. Additionally, the horizontal and vertical electrooculogram and electromyogram (from electrodes at the chin) were obtained. Signals were amplified (BrainAmp, Brain Product) and digitized at a sampling rate of $250 \mathrm{~Hz}$. The EEG was filtered between 0.3 and $35 \mathrm{~Hz}$. Sleep stages were scored offline in 30-sec epochs following standard criteria (Rechtschaffen and Kales 1968). For each subject, we determined sleep latency (with reference to lights off), total sleep time (starting with sleep onset), time spent in different sleep stages, i.e., wake, nonrapid eye movement (NonREM) sleep stages $1,2,3$, and 4 , and REM sleep (in minutes). Slow wave sleep (SWS) was defined by the sum of stage 3 and 4 sleep. Data from one Sleep subject was excluded due to corrupt EEG.

For a more fine-grained analysis of the EEG during sleep, power spectra were calculated using the Brain Vision Analyzer (version 2.0, Brain Products). Following removal of epochs contaminated by visually identified artifacts, Fast Fourier Transformations (0.061- $\mathrm{Hz}$ resolution) with a Hanning window was applied to a 10 -sec data block which was moved in 5-sec steps in time during the respective sleep stage intervals. Average spectra were calculated across the time an individual spent in NonREM sleep (including stage 2, and SWS) and REM sleep, and also separately for the time spent in SWS and stage 2 sleep. Mean power was determined for the 0.5 - to $4-\mathrm{Hz}$ slow wave activity (SWA), the 0.5 - to $1-\mathrm{Hz}$ slow oscillation, 1- to 4-Hz delta, 4- to 8-Hz theta, 9- to 12-Hz slow spindle, and the 12 - to $15-\mathrm{Hz}$ fast spindle frequency bands.

In addition, to determine spindle density (per $30 \mathrm{sec}$ ) and counts, discrete slow and fast spindles were automatically identified during NonREM sleep (including stage 2 and SWS) using a custom-made algorithm (SpindleToolbox, version 1.1) as described previously in Wilhelm et al. (2011). Briefly, for each subject, the slow and fast spindle frequency peaks were visually identified from power spectra in the channels of interest (slow, $11.14 \pm 0.16 \mathrm{~Hz}$; fast, $13.37 \pm 0.13 \mathrm{~Hz}$ ); slow spindles were detected from fronto-central channels (Fz, F3, F4, Cz) and fast spindles from centro-parietal channels (Pz, P3, P4, Cz, C3, C4) according to their respective expected power maxima (Mölle et al. 2011). Then, the root mean square (RMS) of the EEG signal band-passfiltered in the $\pm 1.5-\mathrm{Hz}$ range around the detected spindle peak was calculated for subsequent 0.2 -sec intervals separately for each EEG channel. A spindle was counted when the signal exceeded an individual amplitude threshold of 1.5 standard deviations from the mean RMS in a specific channel for $0.5-3 \mathrm{sec}$.

\section{Statistical analyses}

Statistical testing was done using $[R]$ (64-bit Windows version 2.15.0) (R Core Team 2012). Values are given as mean \pm SEMs.
Pre-tests involved Shapiro-Wilk's test for normality and for group tests, and Levene's test for homoscedasticity. To assess differences between Sleep and Wake groups, we used Student's $t$-test for equal variances and Welch's $t$-test with approximation to the degrees of freedom for unequal variances, when normality was assumed; otherwise we used nonparametric Mann-Whitney rank sum test with either exact $P$-values, or $P$-values that were continuity corrected in normal approximation. Cohen's $d$ and Pearson's $r$ were used to indicate effect size for parametric and nonparametric tests, respectively. Unless otherwise indicated $P$-values are reported uncorrected for multiple comparisons. The significance level was set to $\alpha<0.05$.

\section{Acknowledgments}

We thank Professors B. Pause and C. Jungbluth for sharing their experiences with eye-tracking technology. This study was funded by the Deutsche Forschungsgemeinschaft SFB 654 (Plasticity and Sleep). J.-Y.W. was supported by the Chinese Scholarship Council.

\section{References}

Abel M, Bäuml KH. 2013. Sleep can reduce proactive interference. Memory. doi: $10.1080 / 09658211.2013 .785570$.

Allen TA, Fortin NJ. 2013. The evolution of episodic memory. Proc Natl Acad Sci 110 Suppl 2: $10379-10386$.

Barakat M, Doyon J, Debas K, Vandewalle G, Morin A, Poirier G, Martin N, Lafortune M, Karni A, Ungerleider LG, et al. 2011. Fast and slow spindle involvement in the consolidation of a new motor sequence. Behav Brain Res 217: $117-121$

Cairney SA, Durrant SJ, Musgrove H, Lewis PA. 2011. Sleep and environmental context: Interactive effects for memory. Exp Brain Res 214: $83-92$.

Cheng CY, Yen MY, Lin HY, Hsia WW, Hsu WM. 2004. Association of ocular dominance and anisometropic myopia. Invest Ophthalmol Vis Sci 45: 2856-2860.

Clayton NS, Dickinson A. 1998. Episodic-like memory during cache recovery by scrub jays. Nature 395: 272-274.

Clayton NS, Bussey TJ, Dickinson A. 2003. Can animals recall the past and plan for the future? Nat Rev Neurosci 4: 685-691.

Clemens Z, Mölle M, Eross L, Barsi P, Halasz P, Born J. 2007. Temporal coupling of parahippocampal ripples, sleep spindles and slow oscillations in humans. Brain 130: 2868-2878.

Davis KE, Eacott MJ, Easton A, Gigg J. 2013a. Episodic-like memory is sensitive to both Alzheimer's-like pathological accumulation and normal ageing processes in mice. Behav Brain Res. 254: 73-82.

Davis KE, Easton A, Eacott MJ, Gigg J. 2013b. Episodic-like memory for what-where-which occasion is selectively impaired in the 3xTgAD mouse model of Alzheimer's disease. J Alzheimers Dis 33: 681-698.

Dere E, Kart-Teke E, Huston JP, De Souza Silva MA. 2006. The case for episodic memory in animals. Neurosci Biobehav Rev 30: 1206-1224

Dere E, Pause BM, Pietrowsky R. 2010. Emotion and episodic memory in neuropsychiatric disorders. Behav Brain Res 215: 162-171.

DeVito LM, Eichenbaum H. 2010. Distinct contributions of the hippocampus and medial prefrontal cortex to the "what-wherewhen" components of episodic-like memory in mice. Behav Brain Res 215: $318-325$.

Diekelmann S, Born J. 2010. The memory function of sleep. Nat Rev Neurosci 11: 114-126.

Ebner NC, Riediger M, Lindenberger U. 2010. FACES-A database of facial expressions in young, middle-aged, and older women and men: Development and validation. Behav Res Methods 42: 351-362.

Ennaceur A, Delacour J. 1988. A new one-trial test for neurobiological studies of memory in rats. 1: Behavioral data. Behav Brain Res 31: 47-59.

Ennaceur A, Meliani K. 1992. A new one-trial test for neurobiological studies of memory in rats. III. Spatial vs. non-spatial working memory. Behav Brain Res 51: 83-92.

Frankland PW, Bontempi B. 2005. The organization of recent and remote memories. Nat Rev Neurosci 6: 119-130.

Gais S, Mölle M, Helms K, Born J. 2002. Learning-dependent increases in sleep spindle density. J Neurosci 22: 6830-6834.

Inostroza M, Born J. 2013. Sleep for preserving and transforming episodic memory. Annu Rev Neurosci 36: 79-102.

Inostroza M, Binder S, Born J. 2013a. Sleep-dependency of episodic-like memory consolidation in rats. Behav Brain Res 237: 15-22. 
Inostroza M, Brotons-Mas JR, Laurent F, Cid E, de la Prida LM. 2013b. Specific impairment of "what-where-when" episodic-like memory in experimental models of temporal lobe epilepsy. J Neurosci 33: 17749-17762.

Kart-Teke E, Silva MADS, Huston JP, Dere E. 2006. Wistar rats show episodic-like memory for unique experiences. Neurobiol Learn Mem 85: $173-182$.

Kart-Teke E, Dere E, Brandao ML, Huston JP, De Souza Silva MA. 2007. Reinstatement of episodic-like memory in rats by neurokinin-1 receptor antagonism. Neurobiol Learn Mem 87: 324-331.

Klein SB. 2013. Making the case that episodic recollection is attributable to operations occurring at retrieval rather than to content stored in a dedicated subsystem of long-term memory. Front Behav Neurosci 7: 3. doi: 10.3389 /fnbeh.2013.00003.

Krohne HW, Egloff B, Kohlmann C-W, Tausch A. 1996. Study with a German form of the Positive and Negative Affect Schedule (PANAS). Diagnostica 42: 139-156.

Lewis PA, Durrant SJ. 2011. Overlapping memory replay during sleep builds cognitive schemata. Trends Cogn Sci 15: 343-351.

Lewis PA, Cairney S, Manning L, Critchley HD. 2011. The impact of overnight consolidation upon memory for emotional and neutra encoding contexts. Neuropsychologia 49: 2619-2629.

Li JS, Chao YS. 2008. Electrolytic lesions of dorsal CA3 impair episodic-like memory in rats. Neurobiol Learn Mem 89: 192-198.

Libkuman TM, Stabler CL, Otani H. 2004. Arousal, valence, and memory for detail. Memory 12: 237-247.

Marr D. 1971. Simple memory: A theory for archicortex. Philos Trans R Soc Lond B Biol Sci 262: 23-81.

Mitchell JB, Laiacona J. 1998. The medial frontal cortex and temporal memory: Tests using spontaneous exploratory behaviour in the rat. Behav Brain Res 97: 107-113.

Mölle M, Born J. 2011. Slow oscillations orchestrating fast oscillations and memory consolidation. Prog Brain Res 193: 93-110.

Mölle M, Bergmann TO, Marshall L, Born J. 2011. Fast and slow spindles during the sleep slow oscillation: Disparate coalescence and engagement in memory processing. Sleep 34: 1411-1421.

Ngo HV, Martinetz T, Born J, Mölle M. 2013. Auditory closed-loop stimulation of the sleep slow oscillation enhances memory. Neuron $\mathbf{7 8}$ $545-553$.

Pause BM, Jungbluth C, Adolph D, Pietrowsky R, Dere E. 2010. Induction and measurement of episodic memories in healthy adults. $J$ Neurosci Methods 189: 88-96.

Pause BM, Zlomuzica A, Kinugawa K, Mariani J, Pietrowsky R, Dere E. 2013. Perspectives on episodic-like and episodic memory. Front Behav Neurosci 7: 33. doi: 10.3389/fnbeh.2013.00033.

Penton-Voak IS, Perrett DI, Castles DL, Kobayashi T, Burt DM, Murray LK, Minamisawa R. 1999. Menstrual cycle alters face preference. Nature 399: $741-742$
Place R, Lykken C, Beer Z, Suh J, McHugh TJ, Tonegawa S, Eichenbaum H, Sauvage MM. 2012. NMDA signaling in CA1 mediates selectively the spatial component of episodic memory. Learn Mem 19: $164-169$.

Plihal W, Born J. 1997. Effects of early and late nocturnal sleep on declarative and procedural memory. J Cogn Neurosci 9: 534-547.

Rasch B, Born J. 2013. About sleep's role in memory. Physiol Rev 93: $681-766$.

Rauchs G, Bertran F, Guillery-Girard B, Desgranges B, Kerrouche N, Denise P, Foret J, Eustache F. 2004. Consolidation of strictly episodic memories mainly requires rapid eye movement sleep. Sleep 27: 395-401.

R Core Team. 2012. R: A language and environment for statistical computing. $\mathrm{R}$ Foundation for Statistical Computing, Vienna, Austria.

Rechtschaffen A, Kales A. 1968. A manual of standardized terminology, techniques and scoring system for sleep stages of human subjects. Neurological Information Network, Bethesda, MD.

Spencer RM, Sunm M, Ivry RB. 2006. Sleep-dependent consolidation of contextual learning. Curr Biol 16: 1001-1005.

Squire LR. 1992. Memory and the hippocampus: A synthesis from findings with rats, monkeys, and humans. Psychol Rev 99: 195-231.

Stickgold R. 2005. Sleep-dependent memory consolidation. Nature 437: $1272-1278$

Tamaki M, Matsuoka T, Nittono H, Hori T. 2009. Activation of fast sleep spindles at the premotor cortex and parietal areas contributes to motor learning: A study using sLORETA. Clin Neurophysiol 120: $878-886$.

Tulving E. 1983. Elements of episodic memory. Clarendon Press, Oxford, UK.

Tulving E. 2001. Episodic memory and common sense: How far apart? Philos Trans R Soc Lond B Biol Sci 356: 1505-1515.

Tulving E. 2002. Episodic memory: From mind to brain. Annu Rev Psychol 53: $1-25$.

van der Helm E, Gujar N, Nishida M, Walker MP. 2011. Sleep-dependent facilitation of episodic memory details. PLoS One 6: e27421.

Watson D, Clark LA, Tellegen A. 1988. Development and validation of brief measures of positive and negative affect: The PANAS scales. J Pers Soc Psychol 54: 1063-1070.

Wilhelm I, Diekelmann S, Molzow I, Ayoub A, Mölle M, Born J. 2011. Sleep selectively enhances memory expected to be of future relevance. $J$ Neurosci 31: 1563-1569.

Winocur G, Moscovitch M, Bontempi B. 2010. Memory formation and long-term retention in humans and animals: Convergence towards a transformation account of hippocampal-neocortical interactions. Neuropsychologia 48: 2339-2356.

Received October 9, 2013; accepted in revised form January 28, 2014. 


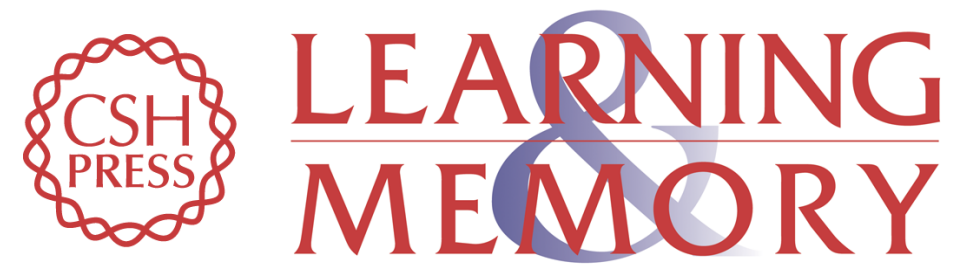

\section{Sleep benefits in parallel implicit and explicit measures of episodic memory}

Frederik D. Weber, Jing-Yi Wang, Jan Born, et al.

Learn. Mem. 2014, 21:

Access the most recent version at doi:10.1101/lm.033530.113

References This article cites 52 articles, 5 of which can be accessed free at: http://learnmem.cshlp.org/content/21/4/190.full.html\#ref-list-1

Creative This article, published in Learning \& Memory, is available under a Creative Commons Commons License (Attribution-NonCommercial 4.0 International), as described at License http://creativecommons.org/licenses/by-nc/4.0/.

Email Alerting Receive free email alerts when new articles cite this article - sign up in the box at the Service top right corner of the article or click here. 